\title{
How to slice: snapshots of Argonaute in action
}

\author{
James S Parker
}

\begin{abstract}
Argonaute is the principal protein component of the mechanisms of RNA silencing, providing anchor sites for the small guide RNA strand and the 'slicer' activity for cleavage of target mRNAs or short passenger RNA strands. Argonaute is the core constituent of the silencing effector complexes RISC (RNA-induced silencing complex) and the RITS complex (RNA-induced initiation of transcriptional gene silencing complex), interacting directly or indirectly with Dicer proteins, R2D2/Loquacious/TRBP and GW182 family proteins in the former, and Chp1 and Tas3 in the latter. In a breakthrough series of papers, Patel et al. provide a set of 'molecular snapshots' of the catalytic cycle of Argonaute, exploiting mismatches and mutants to capture and visualize by X-ray crystallography Argonaute from Thermus thermophilus with guide and target strands at various stages of the silencing process. The structural studies, coupled to structure-directed biochemical analysis, together with other thermodynamic and kinetic studies, provide insights into Argonaute with implications for the mechanisms of RNA silencing in eukaryotes.
\end{abstract}

\section{Introduction}

Genetic and biochemical studies first implicated Argonaute (or Ago) as a key component of the mechanisms of RNA silencing in eukaryotes [1]. Argonaute proteins fall mainly into two subfamilies (Ago and Piwi), defined initially on the basis of sequence similarity [1], with an additional subfamily specific to Caenorhabditis elegans and outliers, which include the prokaryotic Argonautes (eubacterial and archaeal), though the latter category displays some similarity to the Piwi subfamily. Structural studies have revealed the molecular functions of Argonaute, showing that Argonaute is 'Slicer' [2,3], and that it provides anchor sites for the 5' and 3' ends of the guide RNA strand [4-10]. Thus, Argonaute is the principal protein component of RNA silencing. The structural studies also show that three-dimensional Argonaute is roughly a bi-lobal protein, with an $\mathrm{N}$-terminal lobe composed of an $\mathrm{N}$-domain, L1 linker region and PAZ domain (highly mobile) and a C-terminal lobe composed of MID and PIWI domains. Patel et al. now provide the first pictures of full length Argonaute in complex with guide and target strands [11-13], captured at multiple distinct phases of the catalytic cycle, revealing the molecular mechanisms of this slicing machine. As with other structural studies on full length Argonaute, the protein stems from a prokaryote (Thermus thermophilus). This

\footnotetext{
* Correspondence: james.parker@bioch.ox.ac.uk
Department of Biochemistry, University of Oxford, South Parks Road, Oxford

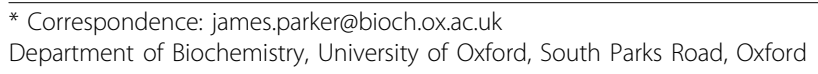
OX1 3QU, UK
}

(c) 2010 Parker; licensee BioMed Central Ltd. This is an Open Access article distributed under the terms of the Creative Commons Attribution License (http://creativecommons.org/licenses/by/2.0), which permits unrestricted use, distribution, and reproduction in any medium, provided the original work is properly cited. are difficult to obtain in the quantities required for $\mathrm{X}$ ray crystallography. Previous studies showed that prokaryotic Argonautes display a preference for a DNA guide strand $[9,14]$ (their in vivo function is still unknown) and, accordingly, Patel and colleagues crystallized the complexes with a DNA guide and RNA targets.

\section{Snapshots of the slicing cycle}

The structures and the stages in the Argonaute cycle that they most closely represent are summarized below and shown in Figure 1:

- The binary complex [11], consisting of Thermus thermophilus Argonaute (TtAgo) and a 21 mer DNA guide strand. The structure is a molecular picture of the substrate-free Argonaute/guide complex, primed for target recognition.

- A mismatched ternary complex [12], consisting of TtAgo, DNA guide and a 20 mer RNA target bearing mismatches to the guide at the 10' and 11' positions (numbered from the 5' end of the guide). With mismatches surrounding the scissile phosphate (between nucleotides $10^{\prime}$ and $11^{\prime}$ ), the structure is representative of a slicing-inactive complex, reminiscent of a microRNA (miRNA)/passenger or miRNA/messenger RNA (mRNA) target complex.

- 12 mer, 15 mer and 19 mer ternary complexes [13], consisting of mutated TtAgo (to inactivate slicing), DNA guide and fully complementary RNA target is because eukaryotic Argonautes from any subfamily 


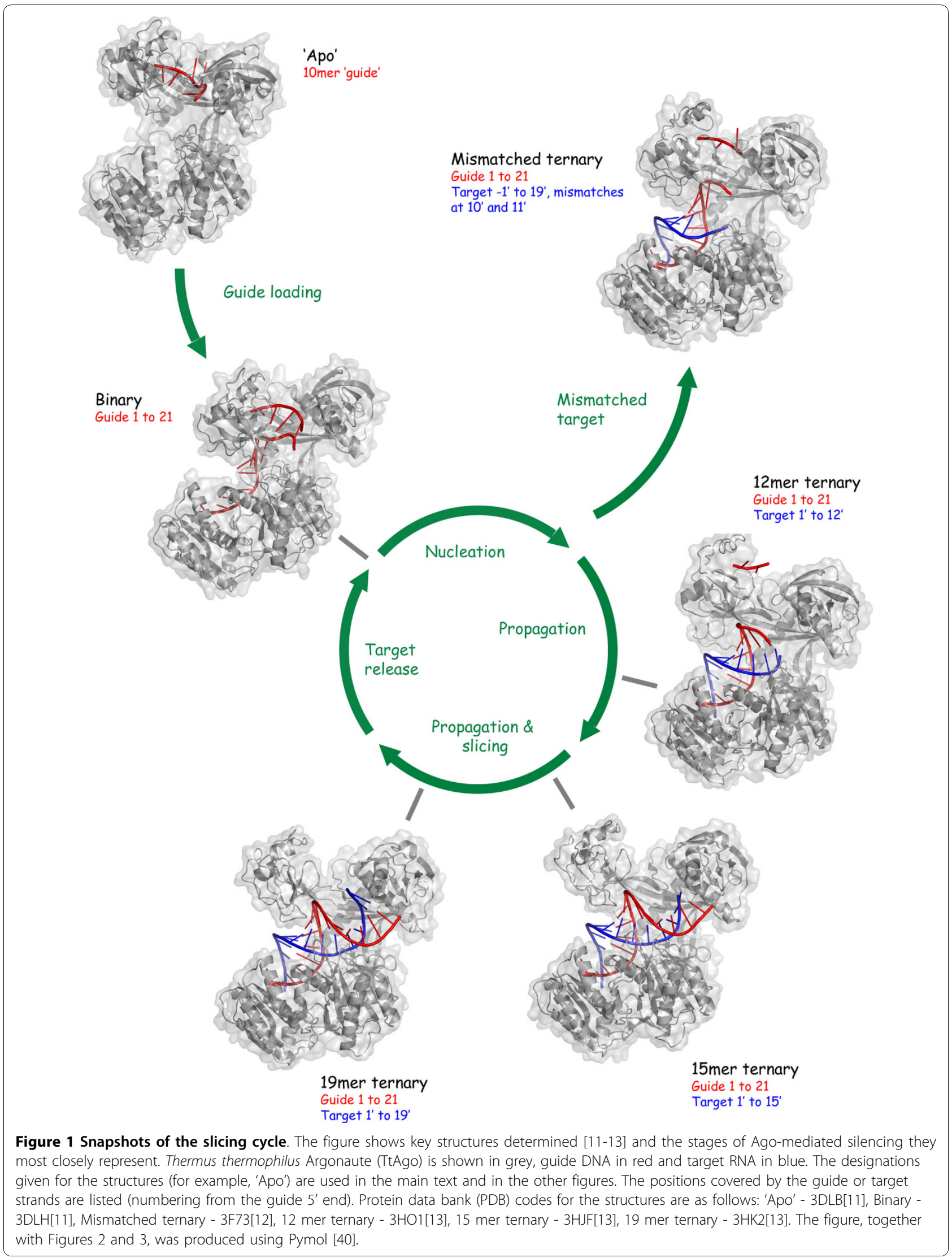


strands of increasing length (12, 15 and 19 nucleotides). It is thought that the target interaction nucleates within positions 2-8 of the guide (the seed region), following which the duplex propagates towards the 3' end of the guide [14-18]. These structures, therefore, provide insight into the nucleation, propagation and slicing stages of 'active' slicer complexes.

- Supplementing these structures, Patel and colleagues also solved a binary complex with a short 10 mer DNA 'guide' [11], providing some insight into the conformation of an apo-TtAgo and, though not displayed in Figure 1, a second independent crystal form of the mismatched ternary complex [13] and unmutated 19 mer ternary complexes obtained in the presence of high concentrations of magnesium [13], elucidated to capture the active slicing geometry of the catalytic site.

\section{Anchoring of the guide in Argonaute $5^{\prime}$ and $3^{\prime}$ end tethering}

Previous structural studies on isolated domains of Argonaute identified highly conserved anchor sites for the 5' and 3' ends of the guide strand [4-10]. The TtAgo structures reveal these to be key anchor sites in the full length protein, defining the orientation of the guide strand within Argonaute. The structures provide a second example of the geometry of the 5' binding pocket (after AfPiwi $[9,10]$ ), at the junction of the MID and PIWI domains, confirming the previously-described configuration involving a metal ion coordinated to the Cterminal carboxylate of the Argonaute polypeptide and the first (5') and third phosphates of the guide strand. Curiously, in TtAgo, an arginine replaces tyrosine in the highly conserved quartet of residues contacting the 5' phosphate (YKQK), a switch so far unique to this protein. Tethering of the 3' end in the PAZ domain, although not a feature of all the complexes (discussed below), mirrors the interactions observed previously in structures involving eukaryotic PAZ domains [4-8].

\section{Support for the two-state model of Ago function}

Despite the multiple anchoring interactions at the 5 ' and 3 ' binding sites, the structures reveal a dynamic cycle of guide end tethering. Remarkably, the structures support directly a previously proposed scheme known as the 'two-state' model [16]. In this model, the 3' end of the guide switches on and off PAZ during the catalytic cycle, being anchored, inaccessibly, in the binary complex and released in a ternary complex. (The 5' end remains fixed.) The model helps to explain the preferential association of the target with the 5 ' section of the guide $[17,19]$. It would also lead to protection of the 3 ' end of the guide when single-stranded in the binary complex, whilst facilitating duplex annealing during the propagation stages of guide/target duplex formation. By comparing the structure of the binary complex with the structures of the complementary 'propagation' complexes containing target strands of increasing length [13], Patel and colleagues show indeed that TtAgo fixes both ends of the guide in the binary complex, and that the 3 ' end of the guide is released in a ternary complex - once a requisite number of base pairs are formed (15 mer ternary complex) (Figure 2A). Presumably, the propagating duplex accumulates sufficient annealing energy to wrest the 3' end of the guide from the binding site in PAZ. It is noteworthy that this model does not apply when the target strand contains mismatches to the guide at positions 10 and 11 (mismatched ternary complex) [12]. In this ternary structure, PAZ retains the guide 3' end (Figure 1).

Base-specificity for the $\mathbf{5}^{\prime}$ nucleotide of the guide

Argonaute proteins are carriers for small RNAs almost irrespective of sequence, which is reflected in the almost complete absence of base-specific contacts in any of the TtAgo complexes. The notable exception is the 5' nucleotide of the guide, which is frequently uridine in miRNAs and Piwi-interacting RNAs (piRNAs) and, strikingly, is capable of directing the sorting of small RNAs into different Argonaute family members in Arabidopsis (whether uridine, adenosine or cytidine) [20-22]. The high-resolution structures of the ternary propagation complexes [13] provide the first insight into how this selectivity could be mediated, through the identification of base-specific contacts between the Ago scaffold and the 5' nucleotide. Asn413 in TtAgo, whose side chain contacts directly the thymine base (DNA guide), is semi-conserved across the Argonaute family (as asparagine, glutamine or threonine). Interestingly, the Arabidopsis Argonautes display unusual diversity at this position, substituting either glutamine (in Ago5), cysteine (in Ago6), or leucine, alanine and valine (other Agos). A more complete picture will, however, require a eukaryotic Ago - guide RNA structure, as the prokarytic Argonautes are substantially diverged and reveal only the most conserved interactions.

\section{Target recognition}

A substantial body of evidence indicates that the primary region within the guide for target recognition, in both small interfering RNA(siRNA) and miRNAmediated silencing, is nucleotides 2 to $7 / 8$ (as measured from the 5' end) [18]. This region, known as the seed sequence [23], provides the specificity in target selection, and a greater portion of the target binding energy $[17,19,24]$. Indeed, in some instances, complementarity over the seed region can be sufficient to mediate silencing [25]. However, within animal miRNAs, where central and 3' complementarity is not required for slicing, a 


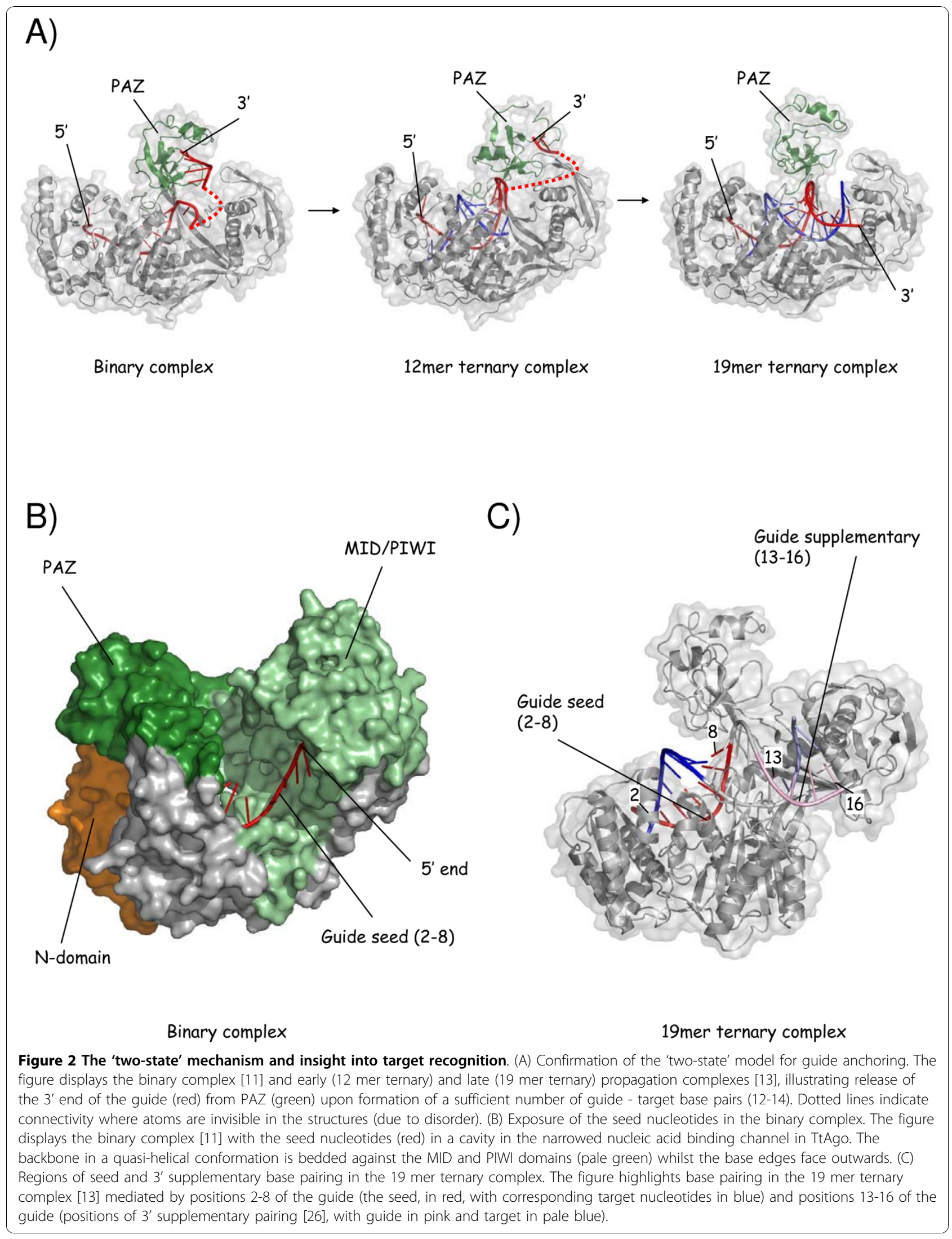


beneficial effect for 3' pairing is still detectable [24-26], particularly between positions 13 and 16 [26], implying a role in binding stability.

What can we learn from the structures about target recognition? The binary complex structure, representing the guide and Argonaute poised to recognize a target as part of, say, RISC or RITS, is very informative [11]. Crucially, the seed nucleotides (2-8), despite being single stranded, arrange in an ordered, quasi-helical arrangement within a canyon in TtAgo, bedded against the wall of the MID/PIWI lobe (Figure 2B). The phosphodiester backbone anchors the seed to the protein, whilst the base edges of nucleotides 2 - 6 face outwards, exposed to the exterior, positioned to capture a target. (Nucleotides 7 and 8 are ordered but partially buried.) By contrast, in the 3 ' half of the guide, nucleotides 12 to 17 are disordered (invisible).

What are the energetic consequences of this arrangement for target recognition? Barford and colleagues have recently provided insight into the energetics of the seed-target nucleation stage of target recognition, employing a technique known as isothermal titration calorimetry (ITC) [27]. This method uses the heat absorbed or released during a binding event to provide highly accurate binding affinities and delineation of the relative contributions of enthalpy and entropy to binding. The group utilized a protein from Archaeoglobus fulgidus composed solely of a MID/PIWI lobe (AfPiwi), together with short RNA and DNA oligonucleotides, to recapitulate the protein/nucleic acid platform over the seed region. Notably, they observe that this platform displays a far higher affinity for a target DNA or RNA strand than is observed for a guide in isolation (an enhancement of up to $\sim 300$-fold). Thus, the tethering of the guide to the MID/PIWI lobe imbues the seed with enhanced binding properties.

One could envisage a number of mechanisms for such enhancement. The protein could make additional contacts to the target strand, supplementing those from the base pairs. Alternatively, the protein could facilitate enhancement directly through the guide strand, either via modulation of the entropy of binding, or through the enthalpic enhancement of the base pair interactions, perhaps through effects on solvent structure. Combined crystallographic and thermodynamic analysis reveals the mechanism [27]: seed-to-target binding is tighter because pre-association of the guide with the protein diminishes the entropy penalty incurred during the interaction (a disorder to order transition). The enhancement does not require any new contacts from AfPiwi to the target [27]. Thus, the pre-ordering or tethering of the guide by the protein directly establishes the enhanced binding site. The structure of the TtAgo binary complex confirms and reinforces the importance of this mechanism. As described previously, the complex displays rigid ordering of the 5' portion of the guide, whilst the 3 ' region is substantially disordered [11]. The asymmetry in ordering is consistent with biochemical studies showing preferential target association with the 5 ' portion of the guide $[17,19]$, supplementing effects that may be derived from accessibility and duplex-compatible structure $[15,16]$. Thus, the structural and thermodynamic studies combine to prove a long-standing inference, that ordering of the seed by Argonaute forms the basis for favoured target recognition [14-18]. Furthermore, because Ago pre-pays some of the entropy penalty for guide/target nucleation, the mechanism links the energetics of guide loading into Ago with those of target recognition.

The structures also provide insight into target recognition fidelity. The TtAgo ternary complexes show that the guide/target duplex forms a continuous A-form-like duplex over the seed region, with numerous contacts from Ago to the phosphodiester backbone of the guide strand but, notably, no hydrogen-bonding contacts to the target strand $[12,13]$. This is compatible with the requirement to retain the guide strand within Ago and, in a multiple-turnover situation $[19,28]$, release the target strand. Furthermore, this asymmetry reflects in the tolerance of TtAgo for bulges in either the guide or target over the seed region. Assays show that a bulge in the guide at position 5 abolishes slicing, whereas a bulge at a similar position in the target has little effect [12]. Presumably, the tight network of interactions from TtAgo which are restraining the guide restrict the capacity for distortion of the guide in the guide/target duplex. This may have implications for our understanding of miRNA target recognition, suggesting that target sites containing seed region bulges (for example, the 5' let-7 site in the lin-41 3' UTR in C. elegans [29]) could be better tolerated than those with seed region deletions (or guide bulges). The selective restraint of one strand would explain the apparent capacity of Argonaute to increase destabilization of mismatches (such as G: $U$ wobbles) within the seed region [24,25,27].

The structures of the ternary complexes provide a starting point for the understanding of the contribution of 3' base pairs to animal microRNA target recognition stability [24-26]. The structure of the complementary 19 mer ternary complex [13] reveals, most unexpectedly, that TtAgo blocks base pairing of a complementary guide-target duplex after position 16 (Figure 2C; discussed further later). Duplex forms only between positions 2 and 16. This may, in part, explain why position 16 describes the 3' limit for significant 3' pairing in miRNA target recognition [26]. The mismatched ternary structure, prima facie more relevant to animal miRNA/ target interactions, shows an alternative and distinct 
conformation for the 3' portion of the guide/target duplex [12]. Here, following mismatches at positions 10 and 11, the guide/target duplex disorders between positions 12 and 19, with PAZ retaining the 3' end of the guide. The significance of 3 ' pairing in this structure, if any, is more difficult to ascertain. It is likely that the two structures (complementary and mismatched) provide examples of two possible conformations of the 3' portion of the guide/target duplex within Ago, adopted according to the individual circumstances of a particular recognition event.

Outside of a particular guide strand footprint, one of the major contributors to animal miRNA target site efficacy is proximity to a second site [26,30], with seed spacings of as little as eight nucleotides resulting in the synergistic enhancement of silencing [26]. One explanation for this effect is cooperative interactions between silencing complexes, but the TtAgo crystal structures provide no obvious basis for this. However, the 3' disordering of the target observed in the mismatched ternary structure [12], despite the presence of complementary base pairs, in principle frees up the target and provides an opportunity for a second silencing complex to interface via a seed sequence at close proximity.

\section{The Slicer catalytic site}

Comparison of the slicer catalytic site in TtAgo between the binary and ternary complexes reveals a highly distorted catalytically incompetent site in the binary complex, transitioning to a catalytically competent form upon annealing of a complementary substrate (12 mer, 15 mer and 19 mer ternary complexes) (Figure 3A). An interesting and unexpected feature is the involvement of two arginine residues (R172 and R548) which in the binary complex appear to stabilize a disruption in the quasi-helical nucleotide stack of the guide between the 10th and 11th nucleotides (that is, at the cleavage locus), resulting in their orthogonal arrangement. In the ternary complexes, by contrast, R548 is displaced, allowing the continuation of an unhindered duplex. However, these residues are almost entirely unconserved at these positions in the Argonaute protein family (apart from in some other prokaryotes), which is puzzling for residues that appear clearly to be mechanistically significant in TtAgo.

Mismatches and mutated catalytic residues, used to capture unsliced ternary complexes, distort the catalytic geometry of the active site and so, in a notable feat of crystallography, Patel and colleagues also obtained diffracting crystals of wild-type TtAgo in the presence of a guide and fully-complementary target [13]. The structures present the catalytic geometry of the slicer site for the first time. The slicer residues (DDD), catalytic magnesium ions (x2) and target RNA strand superimpose closely with the structure of an RNase $\mathrm{H}$ catalytic complex, from Bacillus halodurans [13,31]. Thus, as predicted originally from the fold of the PIWI domain $[2,3]$, slicer employs RNase H-like chemistry to execute slicing of the target, or passenger, RNA strand.

\section{PAZ as an inhibitor of slicing?}

Slicer assays using 3'-truncated target strands (3' relative to the guide), which thereby test the slicing capacity of ternary propagation-equivalent complexes, suggest an interesting and novel mode of slicing regulation within Ago [13]. The assays show that truncation too far in the 3' direction inhibits slicing (at or 5' to position 15), implying that slicing is inhibited during the earlier stages of propagation. This is puzzling when these complexes contain a fully complementary duplex covering the seed and central regions. On the other hand, 3'truncation of the guide down to position 9 does not significantly affect slicing - despite the absence of rigid duplex geometry around the scissile phosphate [12]. The switch in activity in the propagation complexes correlates approximately with a change in conformation observed in the ternary complex structures $(12$ mer and $15 \mathrm{mer}$ ), characterized by the release of the 3' end of the guide from PAZ (Figure 2A). Patel and colleagues suggest, therefore, that perhaps PAZ must release the 3' end of the guide in order for slicing to be allowed [13]. This would represent a switch from an inactive to an active slicing conformation. Tethering of the 3 ' end of the guide could influence slicing through the conformation of Ago and/or the guide/target duplex. Notably for the mechanism of slicing inhibition in animal microRNA complexes, PAZ retains the 3' end of the guide in the mismatched ternary complex. Significantly, this could constitute a key aspect of the mechanism of slicing inhibition in animal microRNA effector complexes.

\section{The $\mathbf{N}$-domain as a duplex wedge?}

The 19 mer ternary structure, representing the most complete propagation complex (positions 1 - 19), reveals formation of a regular A-form guide/target duplex but shows, unexpectedly, that the helix terminates at position 16, blocked head-on by the $\mathrm{N}$-domain of TtAgo [13] (Figure 3B). The remaining nucleotides are invisible but the strands are presumed to be separated, passing on either side of the $\mathrm{N}$-domain. As such, the $\mathrm{N}$-domain functions as a wedge, interceding in the trajectory of the annealed guide/target duplex. This could facilitate recycling after slicing, restricting annealing to six base pairs 3 ' of the scissile phosphate, a mechanism that would be particularly important with longer guide strands (for example, with Piwi-interacting RNAs [piRNAs]). Thus, TtAgo pre-unwinds both ends of the annealed guide/ target duplex: position 1 at the $5^{\prime}$ end of the guide and 


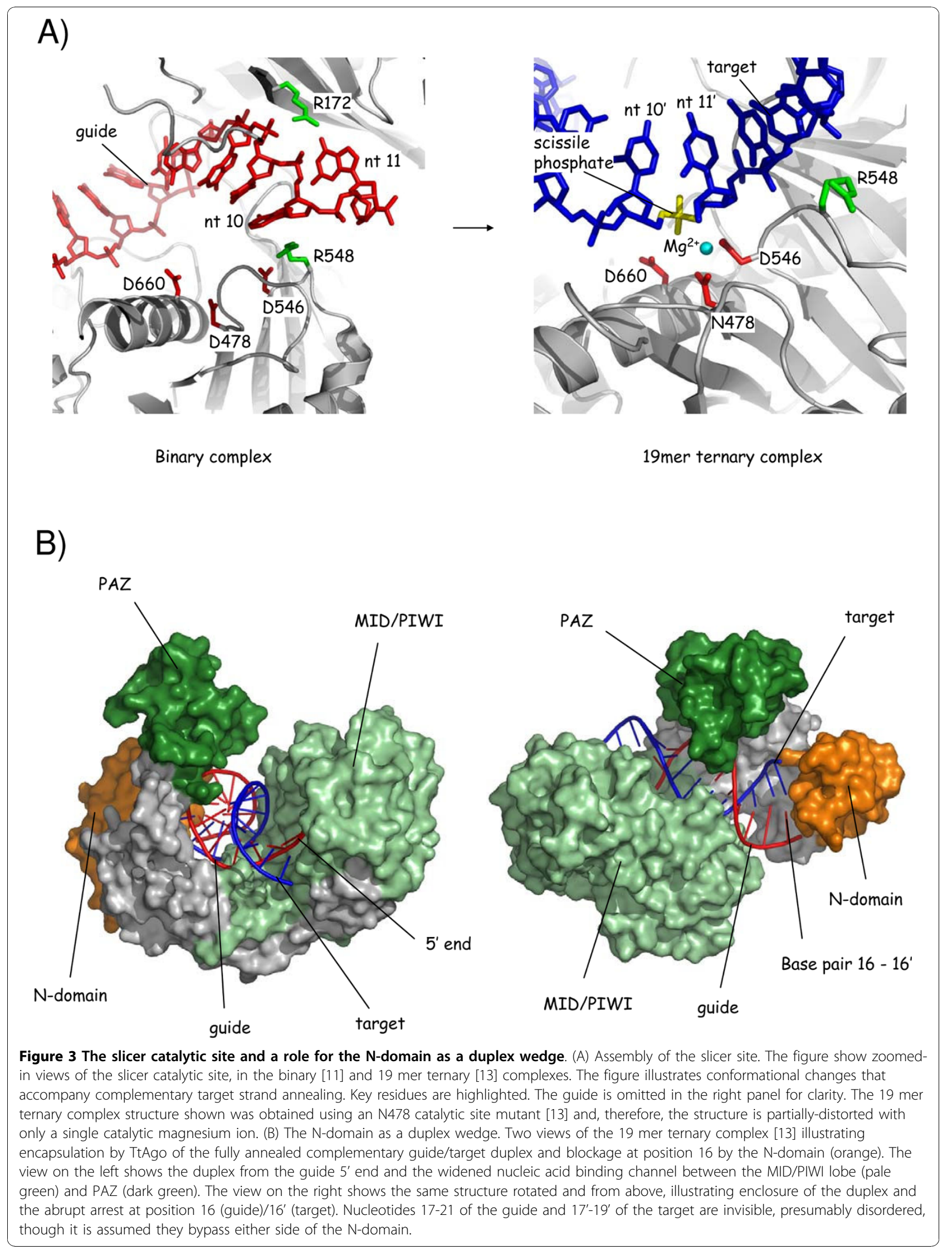


positions 17-21 at the 3' end. An $\alpha$-helix in the $\mathrm{N}$ domain mediates duplex termination, contributing two (unconserved) residues (Y43 and P44) to stack on the end of the duplex; the structure is reminiscent of that which caps and divides the 5 ' end.

\section{Multiple turnover activity}

Eukaryotic RISC and recombinant eukaryotic Argonaute display distinct bi-phasic cleavage kinetics under multiple turnover conditions (excess target strand), with an initial relatively rapid burst of activity followed by a slower 'steady-state' phase $[17,19,32-34]$. This is particularly notable for recombinant eukaryotic Argonaute (human Ago2), which displays very slow steady-state kinetics [33]. The rapid burst of activity probably corresponds to the first single-turnover stage; extrapolation of the steady state rate curve to the ordinate axis yields approximately the concentration of the enzyme in the reaction [17,19,32-34]. This suggests that, under multiple turnover conditions, the recycling stage is the ratelimiting step. Mismatches [19] or competitive blocking [17] at the 3' end of the guide can alleviate this effect, indicating that product release (rather than, say, a regeneration step after product release) is the limiting stage. This may be expected when RNA strand-strand interactions are very tight, with theoretical affinities for $\sim 10$ mer duplexes in the $\mathrm{nM}$ range (dissociation constants). Under some circumstances, adenosine triphosphate (ATP) can also alleviate the effect [19] suggesting that, in vivo, an ATP-assisted enzyme (such as a helicase, or Hsp90 [35-37]) will contribute to recycling. However, at the same time it is clear that RISC as a complex does, overall, substantially weaken the guide/target interaction, because estimated affinities for guide binding to RISC indicate a massively weaker interaction than for the equivalent guide/target interaction in isolation $[17,19]$.

Patel and colleagues show that Thermus thermophilus Argonaute is, in isolation, a multiple turnover enzyme [13]. In contrast to recombinant hAgo2 [33], the enzyme does not appear to display a recycling rate-limiting step, with no evidence for bi-phasic kinetics under multiple turnover conditions (excluding a lag phase) or a substantial difference in rate between single- and multipleturnover conditions. Does this mean that the structural interactions and rearrangements observed upon formation of the slicing-competent complexes reveal the basis for facilitated product release? Unfortunately, this is not so obviously the case, because the TtAgo cleavage assays are conducted at high temperature $\left(75^{\circ} \mathrm{C}\right.$, as the protein stems from a thermophile) which significantly weakens nucleic acid strand interactions, and multiple turnover is conducted using DNA guide and target strands, which in addition interact more weakly than their RNA counterparts. In fact, the theoretical affinities of the sliced DNA cleavage products for the guide, at this temperature, lie between 100 and $400 \mathrm{mM}$ (dissociation constants) $\left(10^{5}\right.$-fold higher than the concentration of nucleic acid in the reactions). Nonetheless, the TtAgo structures provide some clues as to the mechanisms adopted by eukaryotic Argonautes to manage the requirement to function as catalytic enzymes and cleave multiple substrates. First, it is quite striking that there are almost no direct hydrogen-bonding contacts from TtAgo to the target strand across the whole length of the substrate (apart from around the scissile phosphate, which are likely to function to fine-tune positioning of the target with respect to the catalytic residues in the Ago scaffold.) Second, as already discussed, both ends of the formed guide/target duplex are splayed by Ago, which provides a starting point for unwinding. Indeed, one of these free single-stranded ends may be the initial substrate for an ATP-assisted helicase to mediate unwinding. Finally, the extraordinary rearrangements in the Ago scaffold coupled to duplex propagation, evidenced by the pivoting of the Ago domains around an uninterrupted, undistorted guide/target duplex, suggest tensions accumulated and overcome during propagation which may be exploited to eject the sliced target strand, once the duplex is compromised by the central slicing event.

\section{Conclusions and prospects}

The structures of $T$. thermophilus Argonaute in complex with guide and target strands presented by Patel and colleagues provide molecular insight into the central engine of RNA silencing. The structures confirm previously hypothesized mechanisms, including the 'twostate' model for guide tethering, and reveal new ones, such as a potential role for PAZ as an internal inhibitor of slicing, and a role for the $\mathrm{N}$-domain as duplex wedge at the 3' end of the guide. In addition, the structures provide new molecular detail in, for instance, 5 ' nucleotide recognition of the guide, target recognition via the seed sequence and the chemistry of the slicing reaction. These insights are relevant to siRNA, miRNA and piRNA mediated silencing, suggest opportunities for the mutagenesis of eukaryotic Argonautes and provide a molecular basis for the enhancement via chemical modification of reagent and therapeutic siRNAs. Coupled to thermodynamic and kinetic studies from other groups, the structures now provided a detailed mechanistic understanding of the operation of Argonaute. However, a great deal remains to be achieved. These challenges can be viewed in two broad areas. First is the requirement to understand, via structures, the molecular details of eukaryotic Argonautes, from all sub-families. Second 
is the requirement to understand how other proteins interface with Argonaute, including in RISC, RITS and the Sago and piRNA effector complexes. Very recently, the first progress in this area has been reported by Doudna, Nogales, Wang and colleagues [38], and Macrae and colleagues [39], who describe the first electron microscopy reconstructions of human Dicer and the RISC-loading complex. Nonetheless, the prokaryotic Argonautes provide a foundation for this work, whose amenability to high resolution X-ray crystallography have revealed the dynamicity of Ago at the heart of the slicing catalytic cycle.

\section{Abbreviations}

AfPiwi: Archaeoglobus fulgidus Piwi; Ago: Argonaute; ATP: adenosine triphosphate; ITC: isothermal titration calorimetry; mRNA: messenger RNA; miRNA: microRNA; piRNA: piwi-interacting RNA; RISC: RNA-induced silencing complex; RITS [complex]: RNA-induced initiation of transcriptional gene silencing [complex]; siRNA: small interfering RNA; TtAgo: Thermus thermophilus Argonaute.

\section{Acknowledgements}

The author thanks Dinshaw Patel and Yanli Wang for their helpful assistance. This work is supported by the Medical Research Council.

\section{Competing interests}

The author declares that they have no competing interests.

Received: 14 September 2009

Accepted: 12 January 2010 Published: 12 January 2010

\section{References}

1. Carmell MA, Xuan Z, Zhang MQ, Hannon GJ: The Argonaute family: tentacles that reach into RNAi, developmental control, stem cell maintenance, and tumorigenesis. Genes Dev 2002, 16(21):2733-2742.

2. Song JJ, Smith SK, Hannon GJ, Joshua-Tor L: Crystal structure of Argonaute and its implications for RISC slicer activity. Science 2004, 305(5689):1434-1437.

3. Parker JS, Roe SM, Barford D: Crystal structure of a PIWI protein suggests mechanisms for siRNA recognition and slicer activity. Embo J 2004, 23(24):4727-4737.

4. Lingel A, Simon B, Izaurralde E, Sattler M: Structure and nucleic-acid binding of the Drosophila Argonaute 2 PAZ domain. Nature 2003, 426(6965):465-469.

5. Yan KS, Yan S, Faroog A, Han A, Zeng L, Zhou MM: Structure and conserved RNA binding of the PAZ domain. Nature 2003, 426(6965):468474.

6. Song JJ, Liu J, Tolia NH, Schneiderman J, Smith SK, Martienssen RA, Hannon GJ, Joshua-Tor L: The crystal structure of the Argonaute2 PAZ domain reveals an RNA binding motif in RNAi effector complexes. Nat Struct Biol 2003, 10(12):1026-1032

7. Ma JB, Ye K, Patel DJ: Structural basis for overhang-specific small interfering RNA recognition by the PAZ domain. Nature 2004 429(6989):318-322

8. Lingel A, Simon B, Izaurralde $E$, Sattler M: Nucleic acid 3'-end recognition by the Argonaute2 PAZ domain. Nat Struct Mol Biol 2004, 11(6):576-577.

9. Ma JB, Yuan YR, Meister G, Pei Y, Tuschl T, Patel DJ: Structural basis for 5'end-specific recognition of guide RNA by the $A$. fulgidus Piwi protein. Nature 2005, 434(7033):666-670.

10. Parker JS, Roe SM, Barford D: Structural insights into mRNA recognition from a PIWI domain-siRNA guide complex. Nature 2005, 434(7033):663666.

11. Wang $Y$, Sheng G, Juranek S, Tuschl T, Patel DJ: Structure of the guidestrand-containing Argonaute silencing complex. Nature 2008, 456(7219):209-213.
12. Wang $Y$, Juranek S, Li H, Sheng G, Tuschl T, Patel DJ: Structure of an argonaute silencing complex with a seed-containing guide DNA and target RNA duplex. Nature 2008, 456(7224):921-926.

13. Wang $Y$, Juranek S, Li H, Sheng G, Wardle GS, Tuschl T, Patel DJ: Nucleation, propagation and cleavage of target RNAs in Ago silencing complexes. Nature 2009, 461:754-761

14. Yuan YR, Pei Y, Ma JB, Kuryavyi V, Zhadina M, Meister G, Chen HY, Dauter Z Tuschl T, Patel DJ: Crystal structure of $A$. aeolicus Argonaute, a sitespecific DNA-guided endoribonuclease, provides insights into RISC mediated mRNA cleavage. Mol Cell 2005, 19(3):405-419.

15. Filipowicz W: RNAi: the nuts and bolts of the RISC machine. Cell 2005, 122(1):17-20.

16. Tomari Y, Zamore PD: Perspective: machines for RNAi. Genes Dev 2005 19(5):517-529.

17. Ameres SL, Martinez J, Schroeder R: Molecular basis for target RNA recognition and cleavage by human RISC. Cell 2007, 130(1):101-112.

18. Bartel DP: MicroRNAs: target recognition and regulatory functions. Cell 2009, 136(2):215-233

19. Haley B, Zamore PD: Kinetic analysis of the RNAi enzyme complex. Nat Struct Mol Biol 2004, 11(7):599-606.

20. Mi S, Cai T, Hu Y, Chen Y, Hodges E, Ni F, Wu L, Li S, Zhou H, Long C, et al: Sorting of small RNAs into Arabidopsis Argonaute complexes is directed by the $5^{\prime}$ terminal nucleotide. Cell 2008, 133(1):116-127.

21. Montgomery TA, Howell MD, Cuperus JT, Li D, Hansen JE, Alexander AL, Chapman EJ, Fahlgren N, Allen E, Carrington JC: Specificity of ARGONAUTE7-miR390 interaction and dual functionality in TAS3 transacting siRNA formation. Cell 2008, 133(1):128-141.

22. Takeda A, Iwasaki S, Watanabe T, Utsumi M, Watanabe $Y$ : The mechanism selecting the guide strand from small RNA duplexes is different among Argonaute proteins. Plant Cell Physiol 2008, 49(4):493-500.

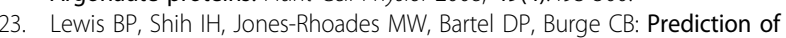
mammalian microRNA targets. Cell 2003, 115(7):787-798.

24. Doench JG, Sharp PA: Specificity of microRNA target selection in translational repression. Genes Dev 2004, 18(5):504-511.

25. Brennecke J, Stark A, Russell RB, Cohen SM: Principles of microRNA-target recognition. PLOS Biol 2005, 3(3):e85.

26. Grimson A, Farh KK, Johnston WK, Garrett-Engele P, Lim LP, Bartel DP: MicroRNA targeting specificity in mammals: determinants beyond seed pairing. Mol Cell 2007, 27(1):91-105

27. Parker JS, Parizotto EA, Wang M, Roe SM, Barford D: Enhancement of the seed-target recognition step in RNA silencing by a PIWI/MID domain protein. Mol Cell 2009, 33(2):204-214.

28. Hutvagner $G$, Zamore PD: A microRNA in a multiple-turnover RNAi enzyme complex. Science 2002, 297(5589):2056-2060.

29. Vella MC, Choi EY, Lin SY, Reinert K, Slack FJ: The C. elegans microRNA let7 binds to imperfect let-7 complementary sites from the lin-41 3'UTR. Genes Dev 2004, 18(2):132-137.

30. Saetrom P, Heale BS, Snove OJr, Aagaard L, Alluin J, Rossi JJ: Distance constraints between microRNA target sites dictate efficacy and cooperativity. Nucleic Acids Res 2007, 35(7):2333-2342.

31. Nowotny M, Gaidamakov SA, Crouch RJ, Yang W: Crystal structures of RNase $\mathrm{H}$ bound to an RNA/DNA hybrid: substrate specificity and metaldependent catalysis. Cell 2005, 121(7):1005-1016.

32. Martinez J, Tuschl T: RISC is a $5^{\prime}$ phosphomonoester-producing RNA endonuclease. Genes Dev 2004, 18(9):975-980.

33. Rivas FV, Tolia NH, Song JJ, Aragon JP, Liu J, Hannon GJ, Joshua-Tor L: Purified Argonaute 2 and an siRNA form recombinant human RISC. Nat Struct Mol Biol 2005, 12(4):340-349.

34. Forstemann K, Horwich MD, Wee L, Tomari Y, Zamore PD: Drosophila microRNAs are sorted into functionally distinct Argonaute complexes after production by dicer-1. Cell 2007, 130(2):287-297.

35. Liu J, Carmell MA, Rivas FV, Marsden CG, Thomson JM, Song JJ, Hammond SM, Joshua-Tor L, Hannon GJ: Argonaute2 is the catalytic engine of mammalian RNAi. Science 2004, 305(5689):1437-1441.

36. Tahbaz N, Kolb FA, Zhang H, Jaronczyk K, Filipowicz W, Hobman TC: Characterization of the interactions between mammalian PAZ PIWI domain proteins and Dicer. EMBO Rep 2004, 5(2):189-194.

37. Pare JM, Tahbaz N, Lopez-Orozco J, LaPointe P, Lasko P, Hobman TC: Hsp90 regulates the function of Argonaute 2 and its recruitment to stress granules and P-bodies. Mol Biol Cell 2009, 20(14):3273-3284. 
38. Wang HW, Noland C, Siridechadilok B, Taylor DW, Ma E, Felderer K, Doudna JA, Nogales E: Structural insights into RNA processing by the human RISC-loading complex. Nat Struct Mol Biol 2009, 16(11):1148-1153.

39. Lau PW, Potter CS, Carragher B, Macrae IJ: Structure of the human DicerTRBP complex by electron microscopy. Structure 2009, 17(10):1326-1332. 40. Pymol home page. http://www.pymol.org/.

doi:10.1186/1758-907X-1-3

Cite this article as: Parker: How to slice: snapshots of Argonaute in action. Silence 2010 1:3.

Publish with Biomed Central and every scientist can read your work free of charge

"BioMed Central will be the most significant development for disseminating the results of biomedical research in our lifetime. " Sir Paul Nurse, Cancer Research UK

Your research papers will be:

- available free of charge to the entire biomedical community

- peer reviewed and published immediately upon acceptance

- cited in PubMed and archived on PubMed Central

- yours - you keep the copyright 